\title{
Fabrication of Magnetic Fluorescent Hollow Microporous Polymer Microspheres
}

\author{
Xiaohu Zou ${ }^{1, a}$, Zhizhi Wei ${ }^{1, b}$, Yan Zhao ${ }^{1, \mathrm{c}}$ and Gaowen Zhang ${ }^{1, d, *}$ \\ ${ }^{1}$ School of Materials and Chemical engineering, Hubei University of Technology, No.28, Nanli Road, \\ Hongshan District, Wuhan City, Hubei Province, China \\ azxhsvx@163.com, bhanyan0409z@163.com, ${ }^{\mathrm{c}} 31913870 @ q q . c o m,{ }^{\mathrm{d}}$ zhgw2003@163.com \\ *corresponding author
}

Keywords: microporous organic polymers, bifunctional microspheres, magnetic nanoparticle, superparamagnetism, fluorescent microspheres

\begin{abstract}
A simple and facile approach of hypercrosslinking was followed to synthesize hollow microporous polymer microspheres (HMPMs) and later incorporated with the magnetic fluorescent bifunctional nanoparticles $\left(\mathrm{Fe}_{3} \mathrm{O}_{4}, \mathrm{CdSe} / \mathrm{ZnS}\right)$ following the in-situ co-precipitation and swelling methodology. The morphology of HMPMs was assessed by high-resolution transmission electron microscope (HRTEM). The magnetic measurements of prepared samples showed superparamagnetism with a maximum magnetization of $24.4 \mathrm{emu} / \mathrm{g}$ at $300 \mathrm{~K}$. Fluorescent absorption measurements revealed emission at $560 \mathrm{~nm}$ suggesting the incorporation of fluorescent QDs. Furthermore the confocal microscope images present the green fluorescence with uniform intensity.
\end{abstract}

\section{Introduction}

The hypercrosslinked polymers (HCPs) have attracted enormous scientific attention because their diverse potential applications in separation ${ }^{[1]}$, heterogeneous catalysis ${ }^{[2]}$, and gas storage ${ }^{[3]}$ due to their large specific surface area, high chemical and thermal stability, low regeneration energy, and synthetic diversity ${ }^{[4,5]}$. Although, extensive work has been done for the synthesis of HCPs, but the functionalization and application have yet to be further improved. As advanced nano-materials, the fluorescent polymer microspheres incorporating quantum dots and superparamagnetic polymer micropheres incorporating magnetic particles were widely studied because of their potential applications in biomedicine areas. A variety of synthetic routes have been developed for the preparation of fluorescent and magnetic microspheres that involve layer-by-layer self-assembly ${ }^{[6,7]}$ where the synthetic protocol is quite tedious, and polymer embedding ${ }^{[8,9]}$ in which polymerization process can easily be affected by fluorescent magnetic nanoparticles. To counter these issues, sufficient attention must be paid to more innovative synthetic techniques so that a major breakthrough will be achieved and its applying value will be improved.

In the present work, hollow microporous polymer microspheres (HMPMs) were prepared based on hypercrosslinking procedure. Firstly, monodispersed silica nano-microspheres were synthesized and modified with silane coupling agent. Secondly, monodispersed silica nano-microspheres were used as template, a series of $\mathrm{SiO}_{2} / \mathrm{PS}-\mathrm{DVB}-\mathrm{AA}$ composite microspheres were synthesized via in situ emulsion polymerization method, which were then hypercrosslinked followed by chemical etching of the sacrificial $\mathrm{SiO}_{2}$ cores to obtain HMPMs. Finally, ferriferrous oxide and $\mathrm{CdSe} / \mathrm{ZnS}$ quantum dots were chosen as magnetic and fluorescent sources respectively to prepare magnetic fluorescent bifunctional HMPMs. On the one hand, the hollow cavity can be used as nanocontainers, which is equipped with high load capacity. Conversely, the porous structure of microporous microspheres can not only accommodate nanocarriers, but also work as a channel for magnetic/fluorescent nanoparticles loading. The magnetic fluorescent hollow microspheres are expected to find their applications in area of biomedicine and heavy metal ion detection.. 


\section{Experimental}

\subsection{Materials}

Styrene (St), tetraethyl orthosilicate (TEOS), 1,2-dichloroethane (DCE), ammonia hydroxide $\left(\mathrm{NH} 3 \cdot \mathrm{H}_{2} \mathrm{O}\right.$ ), sodium dodecyl benzene sulfonate (SDBS), acrylic acid (AA), toluene, Anhydrous ferric chloride $\left(\mathrm{FeCl}_{3}\right), \mathrm{NaHCO}_{3}$, ferric chloride hexahydrate $\left(\mathrm{FeCl}_{3} \cdot 6 \mathrm{H}_{2} \mathrm{O}\right)$, ferrous sulfate heptahydrate $\left(\mathrm{FeSO}_{4} \cdot 7 \mathrm{H}_{2} \mathrm{O}\right)$, isopropanol, methanol and absolute ethanol were analysis grade and purchased from National Medicines Corporation Ltd. of China. Divinylbenzene (DVB, $45 \%$ grade) were purchased from Keda Chemical Co., Ltd. of Shanghai, 3-(trimethoxysilyl)propyl methacrylate (MPS) were purchased from morning silicon Chemical Co. Ltd. of Wuhan, and formaldehyde dimethyl acetal (FDA, Aladdin, $98 \%$ ) were used as received. Potassium persulfate $\left(\mathrm{K}_{2} \mathrm{~S}_{2} \mathrm{O}_{8}\right.$, Fisher) was recrystallized from deionized water prior to drying under reduced pressure. Hydrofluoric acid (HF, $40 \%$ grade, GR) and other reagents of analytical grade were utilized without further purification. CdSe/ZnS quantum dots were purchased from Jiayuan quantum dot Technology Development Co., Ltd. of Wuhan.

\subsection{Synthesis of Hollow Microporous Polymer Microspheres}

In a particular procedure, $1.2 \mathrm{~g}$ of $\mathrm{SiO}_{2}$ were dispersed in $100 \mathrm{~mL}$ water containing SDBS and $\mathrm{NaHCO}_{3}$. The mixture was sonicated under vigorous mechanical stirring. $10 \mathrm{~mL}$ St $3 \mathrm{~mL}$ AA and DVB $(0.025 \mathrm{~mL}, 0.5 \mathrm{~mL}, 0.75 \mathrm{~mL}, 1.0 \mathrm{~mL})$ were added to the mixture. The $\mathrm{K}_{2} \mathrm{~S}_{2} \mathrm{O}_{8}$ was used to initiate emulsion polymerization at $85{ }^{\circ} \mathrm{C}$ under nitrogen protection for $90 \mathrm{~min}$. Then resultants $\left(\mathrm{SiO}_{2} / \mathrm{PS}-\mathrm{DVB}-\mathrm{AA}\right)$ were collected by filtration and dried under vacuum. A total of $20 \mathrm{~mL} \mathrm{DCE}$ was added to the obtained $\mathrm{SiO}_{2} / \mathrm{PS}-\mathrm{DVB}-\mathrm{AA}(1.0 \mathrm{~g})$ for swelling. After stirring for 1 hour, $1.73 \mathrm{~mL}$ FDA were dispersed in and then $3.11 \mathrm{~g} \mathrm{FeCl}_{3}$ was added to the swollen mixture. The original network was formed by stirring at $45^{\circ} \mathrm{C}$ for $5 \mathrm{~h}$, then heated at $80^{\circ} \mathrm{C}$ for $19 \mathrm{~h}$ to get the porous polymer microspheres. The porous polymer microspheres were thoroughly washed with methanol several times, and etched with HF to obtain hollow microporous polymer microspheres (HMPMs) which were further washed with ethanol and dried for later use.

\subsection{Synthesis of Magnetic Mollow Microporous Polymer Microspheres}

$8.115 \mathrm{~g} \mathrm{FeCl}_{3} \cdot 6 \mathrm{H}_{2} \mathrm{O}$ and $5.560 \mathrm{~g} \mathrm{FeSO}_{4} \cdot 7 \mathrm{H}_{2} \mathrm{O}$ (3:2 $\mathrm{M}$ ratio), $0.5 \mathrm{~g}$ HMPMs were added to 200 $\mathrm{mL}$ deionized water and stirred under nitrogen for $8 \mathrm{~h}$, then ammonium hydroxide was added slowly at $50{ }^{\circ} \mathrm{C}$ by using injection pump until the mixture's $\mathrm{pH}$ value reached 9 . Then the suspension was kept at $50^{\circ} \mathrm{C}$ for $1 \mathrm{~h}$. The resultant magnetic HMPMs were centrifuged and washed with deionized water and ethanol several times and dried in a vacuum.

\subsection{Synthesis of Magnetic-fluorescent Hollow Microporous Polymer Microspheres}

The HMPMs $(0.5 \mathrm{~g})$ were swollen in toluene $(4 \mathrm{~mL})$ about $1 \mathrm{~h}, 3 \mathrm{~mL}$ CdSe/ZnS quantum dots $(0.016 \mathrm{~mol} / \mathrm{mL})$ were added into the mixture. After $1 \mathrm{~h}$ of sonication, reaction flask was placed into a multitron shaker about $24 \mathrm{~h}$, the resultant product was centrifuged and exhaustively washed with toluene several times and stored in toluene for later characterization.

\subsection{Characterization}

The morphology and microscope were characterized with high resolution transmission electron microscopy (HRTEM, JEOL) at an accelerating voltage of $200 \mathrm{kV}$. The magnetic properties were acquired on a physics property measurement system PPMS-9T (Quantum Design, US) at $300 \mathrm{~K}$ under an applied magnetic field. The photoluminescent measurement was studied on a IX51 fluorescence spectrophotometer (Olympus, Japan). The excitation and emission spectra of fluorescent HMPMs were acquired on the Cary Eclipse spectrometer (Varian, USA). The absorbance of the sample was studied on the U-3900 UV-vis spectrophotometer (Hitachi, Japan). X-ray powder diffraction (XRD) patterns were studied on (XD-2, Beijing, China) to analyze the crystallographic structure of magnetic HMPMs. 


\section{Results and Discussion}

\subsection{Infrared Spectra Analysis}

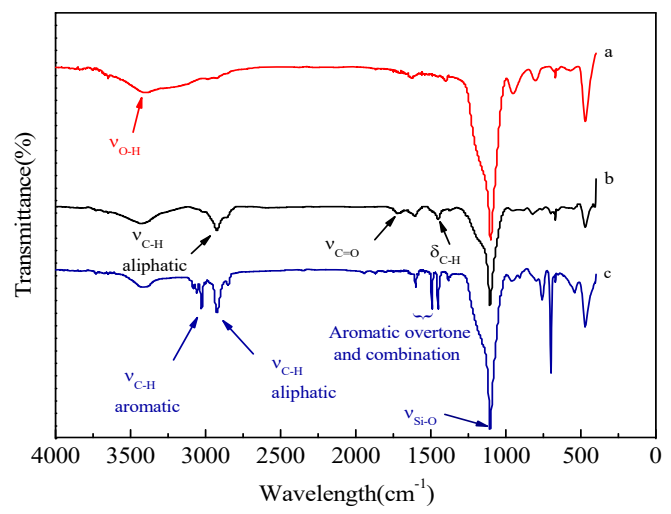

Figure 1 FT-IR spectra of (a) pure silica, (b) modified silica, (c) $\mathrm{SiO}_{2} / \mathrm{PS}-\mathrm{DVB}-\mathrm{AA}$ composite microspheres.

The FTIR spectra of pure silica, modified silica and $\mathrm{SiO}_{2}$ /PS-DVB-AA are given in Fig.1. For the pure silica (Fig.1a), it appeared a broad band at $3410 \mathrm{~cm}^{-1}$ corresponds to O-H stretching vibration $\left(v_{\mathrm{O}-\mathrm{H}}\right)$, a strong absorbance at $1100 \mathrm{~cm}^{-1}$ was attributed to silica $\left(v_{\mathrm{Si}-\mathrm{O}-\mathrm{Si}}\right)$ and the characteristic Si-O group band of pure silica appeared at $469 \mathrm{~cm}^{-1}\left(\delta_{\mathrm{Si}-\mathrm{O}}\right)$. Besides these peaks, the appearance of 2924 $\mathrm{cm}^{-1}\left(v_{\mathrm{C}-\mathrm{H}}\right), 1722 \mathrm{~cm}^{-1}\left(v_{\mathrm{C}=\mathrm{O}}\right), 1607 \mathrm{~cm}^{-1}\left(v_{\mathrm{C}=\mathrm{C}}\right)$ and $1452 \mathrm{~cm}^{-1}\left(\delta_{\mathrm{C}-\mathrm{H}}\right)$ in Fig. $1 \mathrm{~b}$ evidenced the successful modification of nano- $\mathrm{SiO}_{2}$ particles with silane coupling agent $\mathrm{KH}-570$. Fig. 1c shows the IR spectroscopy of $\mathrm{SiO}_{2} / \mathrm{PS}-\mathrm{DVB}-\mathrm{AA}$ composite microsphere. The bands at $3060 \mathrm{~cm}^{-1}, 3026 \mathrm{~cm}^{-1}$ $\left(v_{\mathrm{C}-\mathrm{H}}\right)$ belong to $\mathrm{C}-\mathrm{H}$ stretching vibration of benzene, and the benzene skeleton bands at $1601 \mathrm{~cm}^{-1}$, $1493 \mathrm{~cm}^{-1}, 1452 \mathrm{~cm}^{-1}$ manifested that the modified silica particles were successfully encapsulated in PS-DVB-AA microspheres.

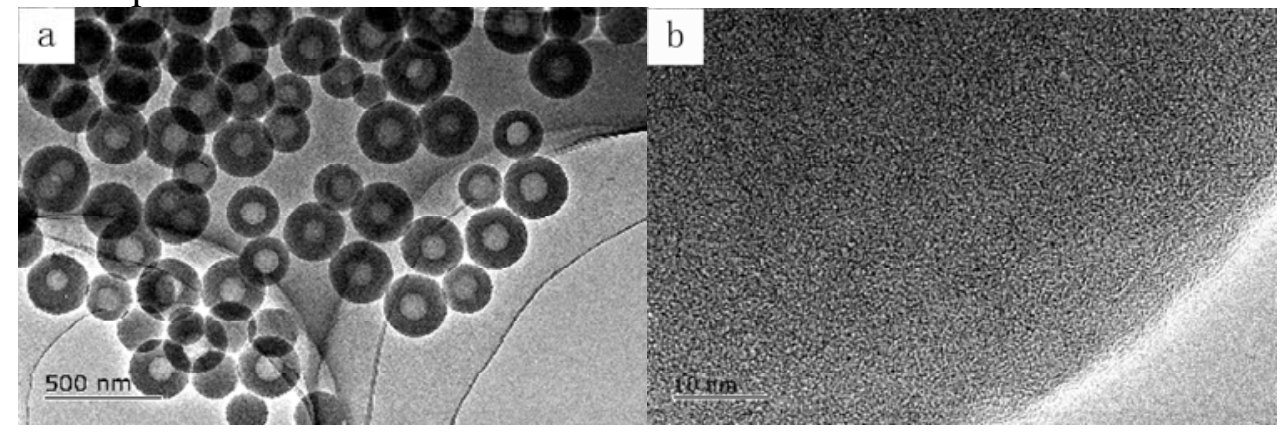

Figure 2 HR-TEM micrographs of hollow microporous polymer microspheres (HMPMs).

a

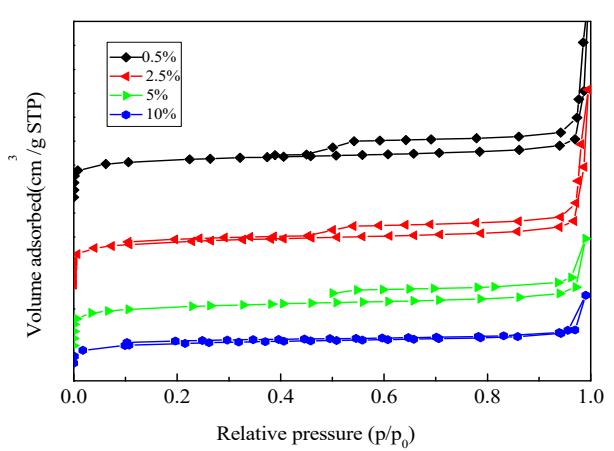

b

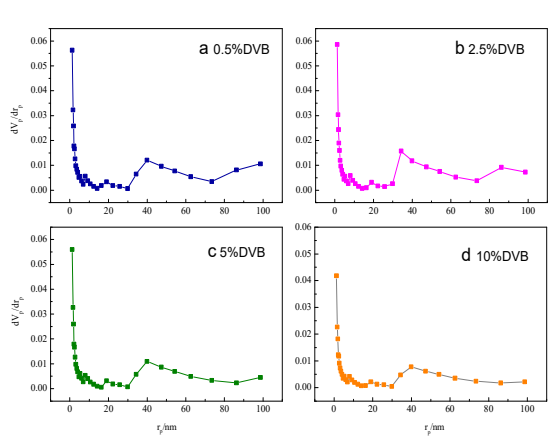

Figure 3 (a) Nitrogen adsorption desorption isotherms at $77 \mathrm{~K}$ of HMPMs, (b) Pore size distribution calculated by BJH at $77 \mathrm{~K}$ of HMPMs. 


\subsection{Morphology of HMPMs}

HRTEM image of HMPMs are shown in Fig.2. Fig.2a clearly depicts the presence of the coreshell structure with a diameter of $200 \mathrm{~nm}$. The presence of dark and white contrast on the surface is ascribed to the existence of extensive micropores (Fig.2b). As shown in Fig.3, all of the polymer networks show Type-I nitrogen sorption isotherms with steep increases at low relative pressure $\left(\mathrm{P} / \mathrm{P}_{0}<0.001\right)$, suggesting that micropores are dominant in these polymer networks. A sharp rise in the nitrogen adsorption isotherms for all networks were also observed at high relative pressures $\left(\mathrm{P} / \mathrm{P}_{0}>0.9\right)$, indicating the presence of some macropores in the polymer networks as well, which are probably due to inter-particle aggregation or void. Significant hysteresis loop was observed at medium and high pressure region $(\mathrm{P} / \mathrm{P} 0=0.42-1.0)$ while $\mathrm{DVB}$ content was $0.5 \%, 2.5 \%$ and $5 \%$. When DVB content was $10 \%$, the hysteresis loop disappeared gradually, which implied that the mesoporous structure is diminishing as corroborated in Fig.4. Thus, it manifests the presence of micropores, mesopores and macropores in the material, simultaneously. The peaks beyond $2 \mathrm{~nm}$ for pore size become weaker, indicating significant reduction of the meso- and macropores by increasing DVB content. All the above results indicate that the increase of DVB content can effectively produce a more uniform and narrower microporous structure.

\subsection{Magnetic Properties Analysis of Magnetic HMPMs}

a

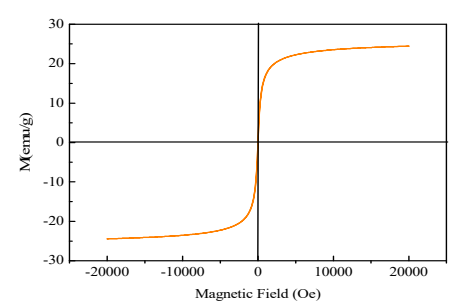

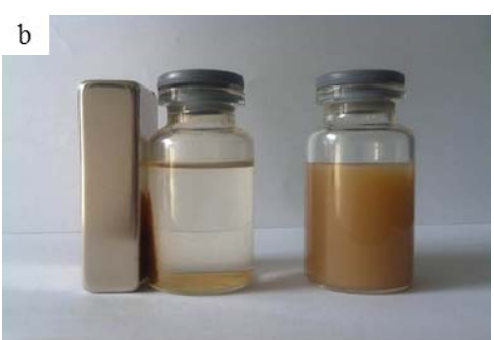

Figure 4 (a) Magnetization curves of magnetic microspheres of in situ precipitation preparation, (b) Image of magnetic HMPMs under external magnetic field.

Upon addition of iron precursor to the carboxyl functionalized microporous microspheres dispersion, iron ions start penetrating into the hollow microspheres, through knitted micropores influenced by the carboxyl groups and ammonia. The $\mathrm{Fe}_{3} \mathrm{O}_{4}$ nanoparticles formed had bigger size than the microspheres pore dimensions, thus the magnetic $\mathrm{Fe}_{3} \mathrm{O}_{4}$ nanoparticles stayed inside of the hollow cavity or in the interporal lacuna.

The magnetic properties of microspheres were monitored using a physics property measurement system PPMS-9T under a maximum applied field of $300 \mathrm{~K}$. Fig.4a illustrates the magnetization saturation of magnetic microspheres is about $24.4 \mathrm{emu} / \mathrm{g}$. Neither remanence nor coercivity in the hysteresis loop was observed suggesting superparamagnetic nature of magnetic microspheres. Fig. $4 \mathrm{~b}$, shows the separation and redispersion process of the magnetic microspheres. On exposure to external magnetic field, the magnetic microspheres were aggregated together leading a clear transparent solution behind, which suggests that magnetic microspheres possess strong magnetic properties.

\subsection{Fluorescence Properties Analysis of Fluorescent HMPMs.}

For the advance studies, magnetic microspheres were incorporated with fluorescent QDs. Fig.5a shows the fluorescence emission spectra of HMPMs under $365 \mathrm{~nm}$ excitation. As shown in Fig.5a, the peak appeared at $560 \mathrm{~nm}$ which suggested the successful loading of fluorescent QDs in the magnetic microspheres. For the better understanding of fluorescent property of HMPMs, the samples were examined under the confocal microscope. The micrograph obtained(Fig.5b) showed the green fluorescence with uniform fluorescence intensity. This green fluorescence is credited to the incorporation of QDs within the microporous structure. 
a

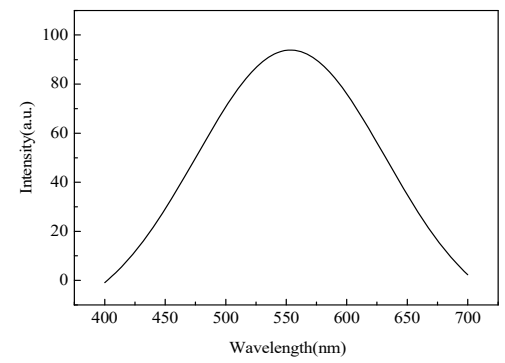

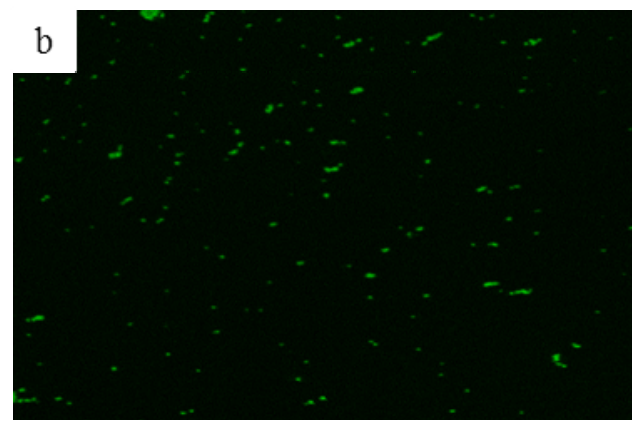

Figure 5 (a) The fluorescence emission spectra of HMPMs under $365 \mathrm{~nm}$ excitation, (b) Confocal fluorescence images of HMPMs (60×objective lens).

\subsection{QDs' Content in Different HMPMs}

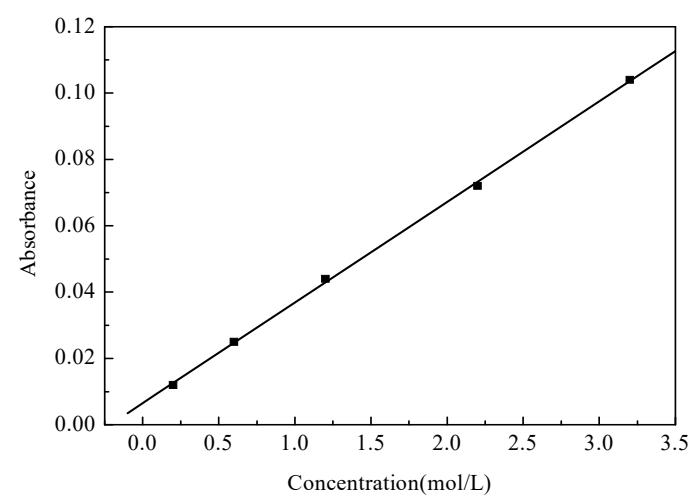

Figure 6 The standard curve of $\mathrm{CdSe} / \mathrm{ZnS}$ concentration.

Standard toluene solution with QDs' concentration of $0.2 \times 10^{-6}, 0.6 \times 10^{-6}, 1.2 \times 10^{-6}, 2.2 \times 10^{-6}$, $3.2 \times 10^{-6} \mathrm{~mol} / \mathrm{L}$ were prepared. And the absorbance of different concentrations of QDs in toluene was measured. As shown in Fig.6, the relationship between the absorbance and the concentration was linear. UV visible spectrophotometer (UV-vis) tested the absorbance of supernatant of HMPMs with different pore size distribution (sample a, b, c, d) under the same concentration of QDs. On the basis of standard curve, the total content of QDs can be calculated. Then, by subtracting the QDs in supernatant from the total, the QDs' loading capacity of magnetic-fluorescent HMPMs were obtained.

\section{Conclusions}

In this report, porous polymer microspheres were prepared via hypercrosslinking procedure, and with such porous microspheres as templates, the magnetic fluorescent bifunctional HMPMs were prepared through in-situ co-precipitation and swelling method with ferriferrous oxide and $\mathrm{CdSe} / \mathrm{ZnS}$ quantum dots as magnetic and fluorescent sources respectively. It has been demonstrated that the magnetic fluorescent HMPMs are superparamagnetic, whose saturation magnetizations are up to $24.4 \mathrm{eum} / \mathrm{g}$. Fluorescence microscopy observes that magnetic-fluorescent HMPMs present green fluorescence. Fluorescent photometer finds that under excitation light of $365 \mathrm{~nm}$, the fluorescence of magnetic fluorescent HMPMs are concentrated at abount $560 \mathrm{~nm}$. When toluene as swelling agent, oil-soluble QDs can be better loaded into HMPMs. Magnetic fluorescent bifunctional HMPMs can achieve magnetic separation and fluorescence tracer simultaneously, it is expected to be applied in area of biomedicine and heavy metal ion detection.

\section{References}

[1]. Sugiyama, T., Asakura, Y., Uda, T., Shiozaki, T., Enokida, Y. and Yamamoto, I. (2006) Present 
status of hydrogen isotope separation by CECE process at the NIFS. fusion engineering and design, 81, 833-838.

[2]. Du, X., Sun, Y., Tan, B., Teng, Q., Yao, X., Su, C. and Wang, W. (2010) Tröger's basefunctionalised organic nanoporous polymer for heterogeneous catalysis. chemical communications, 46, 970-972.

[3]. McKeown, N.B., Budd, P.M. and Book, D. (2007) Microporous polymers as potential hydrogen storage materials. Macromol. rapid communications in mass spectrometry, 28, 995-1002.

[4]. Yang, X., Yu, M., Zhao, Y., Zhang, C., Wang, X. and Jiang, J.X. (2014) Hypercrosslinked microporous polymers based on carbazole for gas storage and separation. RSC Advances, 4, 6105161055.

[5]. Egeblad, K., Christensen, C.H., Kustova, M. and Christensen, C.H. (2008) Templating Mesoporous Zeolites $\uparrow$. chemistry of materials, 20, 946-960.

[6]. Wilson, R., Spiller, D.G., Prior, I.A., Bhattd, R. and Hutchinsone, A. (2007) Magnetic microspheres encoded with photoluminescent quantum dots for multiplexed detection. Journal of marerials chemistry a, 17, 4400-4406.

[7]. Salgueiriño - Maceira, V., Correa - Duarte, M.A., Spasova, M., Liz-Marzán, L.M. and Farle, M. (2006) Composite silica spheres with magnetic and luminescent functionalities. advanced functional materials, 16, 509-514.

[8]. Xie, H.Y., Zuo, C., Liu, Y., Zhang, Z.L., Pang, D.W., Li, X.L., Gong, J.P., Dickinson, C. and Zhou, W. (2005) Cell - Targeting Multifunctional Nanospheres with both Fluorescence and Magnetism. Small, 1, 506-509.

[9]. Yi, D.K., Selvan, S.T., Lee, S.S., Papaefthymiou, G.C., Kundaliya, D. and Ying, J.Y. (2005) Silica-coated nanocomposites of magnetic nanoparticles and quantum dots. journal of the american chemical society, 127, 4990-4991. 